\title{
Impact of Environmental Thermal Stimulation on Activation of Hypothalamic Neuronal Nitric Oxide Synthase during the Prenatal Ontogenesis in Muscovy Ducks
}

\author{
Valery Dunai $^{1,2}$ and Barbara Tzschentke ${ }^{1}$ \\ ${ }^{1}$ Institute of Biology, Humboldt-University of Berlin, Philippstraße 13, 10115 Berlin, Germany \\ ${ }^{2}$ Department of Human Ecology, Belorussian State University, prospect Nezavisimosti 4, 220050, Minsk, Belarus
}

Correspondence should be addressed to Barbara Tzschentke, barbara.tzschentke@rz.hu-berlin.de

Received 30 October 2011; Accepted 11 December 2011

Academic Editor: Richard A. Morrisett

Copyright ( $) 2012$ V. Dunai and B. Tzschentke. This is an open access article distributed under the Creative Commons Attribution License, which permits unrestricted use, distribution, and reproduction in any medium, provided the original work is properly cited.

\begin{abstract}
The aim of the study is to investigate the influence of prenatal temperature stimulation on neuronal NO synthase (nNOS) expression in the anterior hypothalamus of Muscovy duck embryos. Experiments were performed on embryonic day (E) E20, E23, E28, and E33 using histochemistry for identification of the nicotinamide adenine dinucleotide phosphate-diaphorase (NADPHd) as marker of NOS-containing neurons. Until the experiments, all duck embryos were incubated under standard temperature conditions $\left(37.5^{\circ} \mathrm{C}\right)$. During 3 hours before the start of the experiments, one group was incubated at $37.5^{\circ} \mathrm{C}$ (control group), the second was warm-experienced at $39^{\circ} \mathrm{C}$, and the third was cold-experienced at $34^{\circ} \mathrm{C}$. In normal and warm-incubated duck embryos, nNOS activity could be first detected on E23. Particularly, after cold stimulation, a significant increase in nNOS activity was found in all embryos investigated even on day 20. Warm stimulation obviously induces the opposite effect, but at later embryonic age (E33). It can be concluded that probably in late-term bird embryos NO acts as a mediator of the neuronal cold pathway in the anterior hypothalamus, which might be improved by prenatal cold stimulation.
\end{abstract}

\section{Introduction}

In the course of prenatal and early postnatal ontogeny, environmental factors may influence the development of the respective physiological control systems for the entire life period, especially by changes in neural organization and expression of related effector genes $[1,2]$. During early ontogeny, nitric oxide (NO) plays a very important role in the formation of central nervous networks, for example, as a key player that mediated epigenetic mechanisms in developing neurons [3]. NO is involved in neurotransmitter release $[4,5]$ and synaptic plasticity throughout life, for example, [6-9]. NO is produced by activation of nitric oxide synthase (NOS), which converts L-arginine to L-citrulline and NO [10]. The marker for NOS-positive neurons is nicotinamide adenine dinucleotide phosphate-diaphorase (NADPH-d) [11].
In mammals and birds, during different developmental periods, NO plays a crucial role in regulation of various physiological functions, which are mediated by the hypothalamus, like thermoregulation [12-16], water balance [17, 18] and fever [19,20], feeding behaviour [21-25], energy balance [26], and cardiovascular regulation [27-29]. However, in birds under thermoneutral conditions or heat load, for instance, the thermoregulatory effect of NO seems to be different from that in mammals [16], in which central NO mostly induces heat loss responses and finally decreases body temperature [30].

Investigations on localization of NADPH-d in the chicken brain show that the pattern of expression of $\mathrm{NADPH}-\mathrm{d}$, and thus of nNOS, within the avian and mammalian brain might be largely conserved [31]. In the adult chicken, NADPH-d-containing neurons were found in the 
lateral hypothalamus, dorsal hypothalamic area, hypothalamic periventricular nucleus, paraventricular nucleus, and mammillary area [32].

The present study is related to our project on the influence of the prenatal environment on the prenatal and postnatal development of body functions with the thermoregulatory system as an example and the precocial bird as a model. Because of its long incubation time of 35 days, the Muscovy duck embryo fits very good for such investigations. Further, in our research group the early development of neuronal and peripheral mechanisms of the thermoregulatory system as well as other body functions was intensively investigated in the Muscovy duck [33-37]. At the end of incubation in the Muscovy duck, hypothalamic neuronal thermoregulatory mechanisms are well developed [34]. Further, changes in the prenatal thermal environment may stimulate the development of central thermoregulation with long-term influence when applied during critical developmental periods (epigenetic temperature adaptation) $[1,35,38,39]$. We assume a crucial role of $\mathrm{NO}$ in environmental-induced neuronal plasticity also in the birds' thermoregulatory network during different developmental periods. Related to this hypothesis, the aim of the actual study is to investigate the influence of prenatal temperature stimulation on nNOS expression in the preoptic area of the anterior hypothalamus (PO/AH) of Muscovy duck embryos during second half of incubation using histochemistry.

\section{Material and Methods}

2.1. Incubation of Duck Embryos. In the Muscovy duck the total incubation time comprises 35 days. Experiments were carried out in Muscovy ducks during second half of incubation on the embryonic day of 20 (E20), 23 (E23), 28 (E28), and 33 (E33). Our previous experiments showed that during this incubation window in ducks and also other poultry species a dramatic development of physiological mechanisms occurs. Further, during the last days of incubation the thermoregulatory system develops from an open loop system without feedback mechanisms into a closed feedback control system $[1,33,36,37]$. During this period the duck embryos are more and more able to react on environmental influences nearly in an appropriate way. The eggs were incubated at $37.5^{\circ} \mathrm{C}$ and relative humidity of $60 \%$ (setter) and $80 \%$ (hatcher, from E28 until hatching). These conditions are common in artificial duck incubation. On the day of the experiment, three groups were formed. One group was incubated until the experiments at $37.5^{\circ} \mathrm{C}$ (control group). The second group was acute warm experienced at $39^{\circ} \mathrm{C}$ (warm group) and the third was acute cold experienced at $34^{\circ} \mathrm{C}$ (cold group) during 3 hours before the start of the experiments. It has to be noted that the stronger cold stimulation in comparison to the warm stimulation is necessary to induce changes in nNOS because of the high cold tolerance of bird embryos [40,41].

The following experimental procedures were performed according to the European Community regulations and the German Low for Animal Protection.
TABLE 1: Number of embryos investigated in each age group (E20 to E33) under control conditions $\left(37.5^{\circ} \mathrm{C}\right)$, acute cold stimulation $\left(34^{\circ} \mathrm{C}\right.$ for 3 hours before experiment), and acute warm stimulation $\left(39^{\circ} \mathrm{C}\right.$ for 3 hours before experiment).

\begin{tabular}{lcccc}
\hline \multirow{2}{*}{ Applied temperature } & \multicolumn{5}{c}{ Age of the embryos (E) in days } \\
& E20 & E23 & E28 & E33 \\
\hline $37^{\circ} \mathrm{C}$ & 6 & 6 & 6 & 6 \\
$39^{\circ} \mathrm{C}$ & 6 & 6 & 6 & 6 \\
$34^{\circ} \mathrm{C}$ & 12 & 8 & 6 & 6 \\
\hline
\end{tabular}

2.2. nNOS Histochemistry. In the present study the nNOS activity was investigated in brain slices of the anterior hypothalamus from 80 Muscovy duck embryos using histochemistry. Table 1 presents the number of embryos used for the experiments in each age group between E20 and E33.

Because nNOS and NADPH-d show similar biochemical features, in the present work the histochemical method of identification of the NADPH-d-containing neurons developed by Scherer-Singler and coworker [42] and modified by Hope and Vincent [43] was used.

The embryos were extracted from the eggshell and decapitated. Brains from the embryos were removed and fixed for a period of 90 minutes in $4 \%$ paraformaldehyde in phosphate buffer $(0.1 \mathrm{M}, \mathrm{pH} 7.4)$ according to Matsumoto and co-worker [44]. The brains were washed six times for 30 minutes at $4^{\circ} \mathrm{C}$ in $0.1 \mathrm{M}(\mathrm{pH}$ 8.0) Tris- $\mathrm{HCl}$ solution (Tris- $\mathrm{HCl})$. Later the brains were subjected to incubation in sucrose solutions of $10 \%$ and $25 \%$ in Tris- $\mathrm{HCl}$ for 1.5 and 12 hours, respectively.

Serial brain slices $(25 \mu \mathrm{m})$ were made using a cryostate (Leica Microsystems, Wetzlar). The slices containing the anterior hypothalamic region were attached to chromium gelatine-coated micro slides and washed in $0.1 \mathrm{M}$ Tris- $\mathrm{HCl}$ for $5 \mathrm{~min}$. Later they were incubated for $1-2 \mathrm{~h}$ in a solution containing NADPH-d $(1 \mathrm{mM})$, nitro blue tetrazolium $(0.5 \mathrm{mM})$, Triton X-100 (0.3\%), and dicumarol $(1 \mathrm{mM})$ in Tris- $\mathrm{HCl}$ at $22^{\circ} \mathrm{C}$. Then slices were washed again in Tris$\mathrm{HCl}$ solution for 5 minutes, dehydrated in ethanol, and covered with histokitt and glass coverslips. All chemicals used for the histochemistry were obtained from Sigma-Aldrich, Germany.

2.3. Data Analysis. For analysis, light microscopy and digital photography at a magnification of 40 fold (Zeiss Axioskop II, Zeiss AxioCam HRc) were used. NADPH-d (NOS)-positive neurons were counted in the anterior hypothalamus. Therefore, a rectangle mask $(1000 \mu \mathrm{m} \times 1000 \mu \mathrm{m}$, subdivided into 100 squares of $100 \mu \mathrm{m} \times 100 \mu \mathrm{m})$ was placed near the third ventricle and the nucleus anterior medialis hypothalami above the optic chiasm (Figure 1). Stereotaxic data of the adult chicken brain were taken from Kuenzel and Masson [46] and proportionally adapted for the appliance in the embryonic duck brain. In relation to the age of the embryos, NOSpositive-neurons were counted in 4 to 8 brain slices, which contain the anterior hypothalamus with the preoptic area of the anterior hypothalamus (PO/AH). 


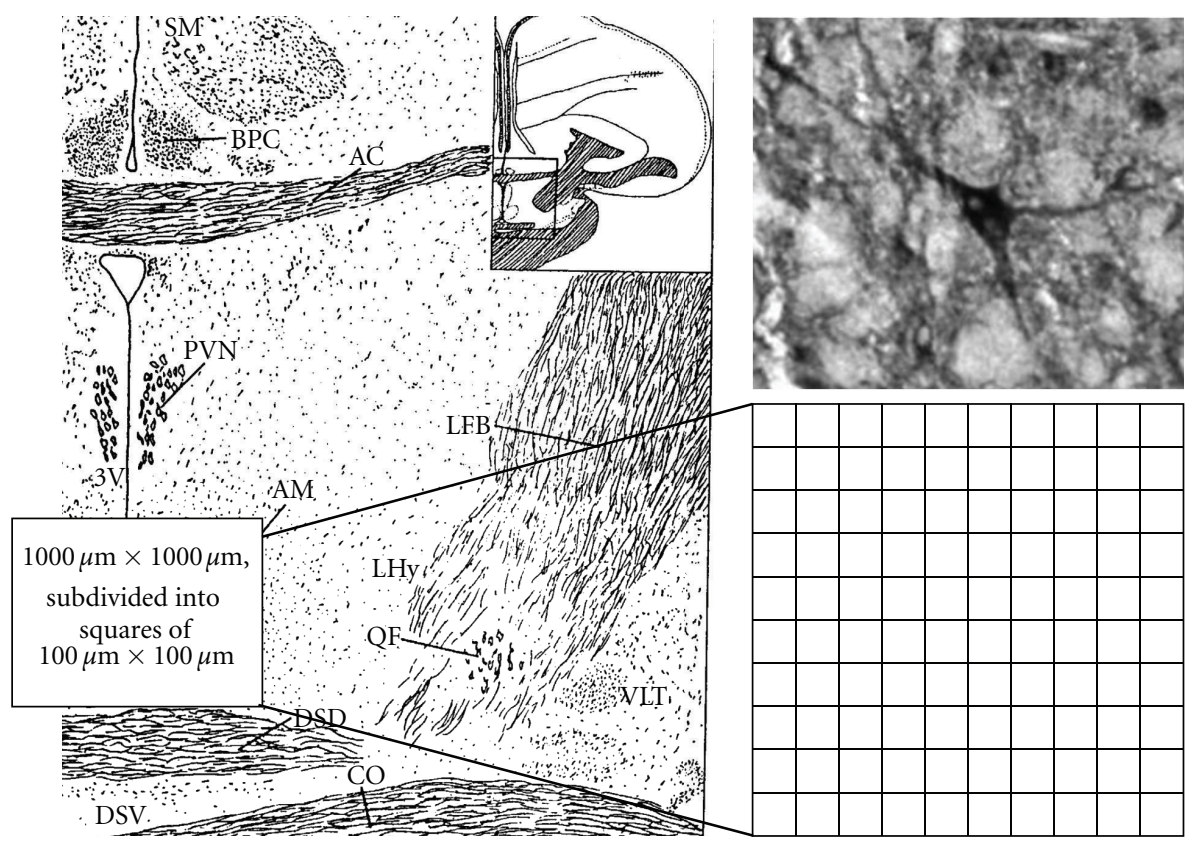

FIGURE 1: Left: anterior hypothalamus with the preoptic area after Kuenzel and Van Tienhoven [45], where the investigations were carried out (AC: Commissura anterior, PVN: Nuc. paraventricularis, 3V: third ventricle, AM: Nuc. anterior medialis hypothalami, CO: Chiasma opticum, LHy: lateral hypothalamic area, and FB: forebrain bundle). Right: typical examples of nNOS expression (magnification $\times 100)$ in a Muscovy duck embryo on E 23. nNOS positive Neurons were counted in a defined area, which was divided into 100 rectangles of $100 \times 100 \mu \mathrm{m}$.

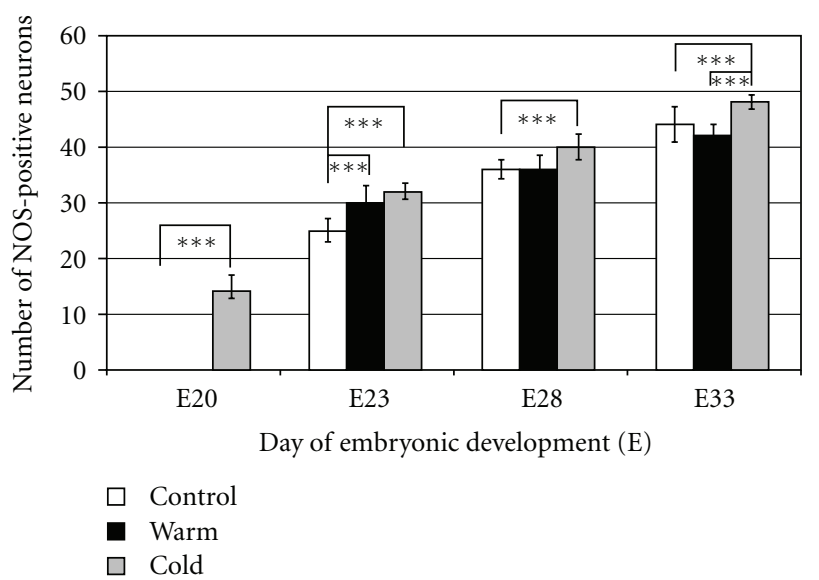

FIGURE 2: Hypothalamic nNOS expression in 20- to 33-days-old Muscovy duck embryos under different temperature treatments (control: constant $37.5^{\circ} \mathrm{C}$, warm: $3 \mathrm{~h} 39^{\circ} \mathrm{C}$, cold: $3 \mathrm{~h} 34^{\circ} \mathrm{C}$ before start of the experiments). Each column represents mean values \pm SD of $100(100 \times 100 \mu \mathrm{m})$ rectangle fields from $4-8$ brain slices of each experimental group. Investigations were carried out in 80 embryos (see Table 1). Asterisks represent significance at the level of $P<0.001$.

Data were expressed as mean $\pm \mathrm{SD}$. The mean values were calculated over all of the 100 counted $100 \mu \mathrm{m}$ squares of the used rectangular mask in all brain slices (4-8), which were prepared from each duck embryo investigated (number of investigated duck embryos see Table 1)
Normal distribution of the data was tested using the Kolmogoroff-Smirnoff Test. The influence of temperature stimulation on nNOS expression in comparison to the control group was tested with Student's $t$-test for independent samples (if normal distributed) or using the MannWhitney $U$-test (if not normal distributed). The influence of embryonic age on nNOS expression was tested using the nonparametric Kruskal-Wallis test. Significance level was set at $P<0.05$.

\section{Results}

In all experimental series (control, cold, and warm stimulated group), a high significant $(P<0.001)$ increase in nNOS activity was found with increasing embryonic age. But on E20, in the control group as well as the warm stimulated group no nNOS-positive neurons were found; nNOS activity could be only observed in the cold stimulated group. Also in older embryos, the highest nNOS activity was obtained after $3 \mathrm{~h}$ cold stimulation in all age groups investigated (Figure 2). Between the control group and the cold stimulated group, the increase in nNOS activity was always of high significance $(P<0.001)$. A $3 \mathrm{~h}$ warm stimulation did not influence nNOS activity on E20 and E28. But a significant influence of warm stimulation on nNOS activity was found on E23 and E33. However, in comparison to the control group on E23 warm stimulation induced a statistically significant $(P<0.001)$ increase and on E33 a decrease (but not statistically significant) in the number of nNOS-positive neurons (Figure 2). 
In summary, a clear influence on nNOS activity was shown after cold stimulation in all age groups of the duck embryos investigated, and always a high significant increase in nNOS was induced by the cold experience.

\section{Discussion}

In precocial birds, like ducks and chicken, the development of body functions starts early during embryogenesis. At the end of incubation, peripheral and central nervous thermoregulatory mechanisms, as well as other physiological functions, are well developed [36, 47]. Finally, the late-term embryo is able to react on changes in incubation temperature. If incubation temperature is increasing, Muscovy duck and chicken embryos react with increasing blood flow, activation of respiration and decrease in heat production [37, 48, 49]. Even under cold load, late-term poultry embryos are not able to keep body temperature constant, endothermic reactions occur when incubation temperature and body temperature are decreasing $[37,50]$. It results in non or very small decrease in oxygen consumption. Under moderate cold load in Muscovy duck embryos, such endothermic counterreactions were already found on E22 [48]. Besides peripheral thermoregulatory mechanisms, a profound development of the neuronal structures in the anterior hypothalamus of Muscovy duck embryos during the second half of incubation corresponds to the electrophysiological findings. In Muscovy duck embryos during extracellular single neuronal recordings, thermosensitive $\mathrm{PO} / \mathrm{AH}$ neurons were found on E22 and E23 [38], which show characteristics similar to the hatching [51] and posthatching period [34], growth period (own experiments, unpublished) and adult birds [52] as well as in mammals [53]. Our hypothesis is that early environmental stimulation of body functions improves their maturation and reactivity to environmental variations ("training effect") during the perinatal period [33]. In chicken, for instance, short-term temperature "training" during the last 4 days of incubation improved vitality of hatched chicken and the posthatching growth [54]. Further, prenatal temperature manipulations may have long-term effects on the posthatching development and adaptability of body functions. In poultry, moderate chronic increase or decrease in incubation temperature during the last days of embryonic development induced postnatal changes in peripheral (e.g., thermoregulatory heat production, preferred ambient temperature, body temperature) and central nervous (e.g., neuronal hypothalamic thermosensitivity) mechanisms of thermoregulation, which are related to warm or cold adaptation [35, 55]. For instance, in 1-day-old Muscovy ducklings, which were long-term cold-incubated $\left(34.5^{\circ} \mathrm{C}\right.$, E28 up to hatching), the thermoregulatory heat production under $1 \mathrm{~h}$ cold load of $10^{\circ} \mathrm{C}$ was significantly higher than in the normal incubated control group. These birds were able to keep their body temperature constant. The present investigations reveal that short-term low temperature application stimulates the activity of nNOS in all age groups investigated. Further, in comparison with warm stimulated embryos and the normal incubated control group, in shortterm cold stimulated embryos nNOS activity was already detectable in an earlier stage of development. Both, the activation of nNOS on an earlier stage of development and the increasing expression of nNOS particularly after cold load in all other age groups investigated lead to the hypothesis that probably in bird embryos $\mathrm{NO}$ acts as a mediator of the neuronal cold pathway in the anterior hypothalamus. In adult mammals, the lateral hypothalamic area via the forebrain bundle controls different autonomic effectors of cold defense or heat conservation, for instance skin blood flow [56, 57], muscle shivering [58] and nonshivering thermogenesis in coordination with regulation of food intake [59]. The importance of NOS in cold-induced thermogenesis was described in rats. In cold environment systemic NOS inhibition by L-NAME injection induced a prolonged fall in body temperature, obvious by prevention of metabolic cold defense [60]. In rat pups aged between days 1 and 6, L-NAME applications abolished the oxygen consumption response to cold and reduced the metabolic cold defense in 10- to 11-day-old pups [14]. In opposite, in mammals under heat load [30], exercise [61], or fever [62] NO manly activates heat loss mechanisms. But in birds also under these conditions the role of NO seems to be different from that observed in mammals. In 5-days-old chicks under thermoneutral and heat stress conditions intramuscular as well as intracerebroventricular injections of NOS inhibitor LNAME decreased body temperature and thus inhibited heat stress-induced hyperthermia [16].

However, in bird embryos, peripheral NO probably acts as a signal indicating the necessity for rewarming of the embryos [63]. NO emission was found mainly via the chorioallantoic membrane in these studies made on 18 bird species. Ar and coworkers [63] speculated that NO emission from eggs may carry a "message" from the embryo to the incubating parents and vice versa. The results from our study, which show a significant lower nNOS activity after shortterm warm-load in comparison with the cold experienced group as well as the control group on E33, support the hypothesis that nNOS activity is a central (and obviously also peripheral) cold signal.

It is interesting that, in our study, in younger embryos short-term warm stimulation induced different changes in hypothalamic nNOS activity. Compared with the control on E23, an increase in nNOS was observed, too, and on E28 a warm load induced no change in nNOS. Only on E33 shortterm warm- and cold-load caused opposite changes in nNOS compared with the control group. These different effects of short-term warm load on nNOS activity may be related to the peculiarity of early development of body functions that acute changes in environmental conditions induce as a rule, first uncoordinated and immediately nonadaptive reactions. Later the uncoordinated (immediately) nonadaptive reactions change into coordinated (adaptive) reactions obviously with the development of feedback mechanisms of the respective system $[1,35,36]$. For instance, experiments at the end of incubation time in chicken embryos revealed first proximate non-adaptive and later adaptive reactions with respect to the influence of cooling and warming on blood flow in the vessels of chorioallantoic membrane. In chicken embryos, the blood flow increased or decreased while warming or 
cooling on E15 until E19 (proximate non-adaptive). After this period, the reaction became proximate adaptive; on E20 and E21, the blood flow in the chorioallantoic membrane increased during warming and decreased during cooling, as expected. Similar changes in the blood flow during cooling were also found in Muscovy duck embryos at the end of incubation. However, in Muscovy duck embryos under warming proximate non-adaptive reactions persisted until day 34 of incubation [36].

In conclusion, the results show that short-term prenatal cold-load may stimulate nNOS activity in the anterior hypothalamus of Muscovy duck embryos during the entire investigated developmental period between E20 and E33. Warm stimulation obviously induces the opposite effect, but it starts at later embryonic age (E33). In younger duck embryos warm stimulation causes proximate nonadaptive reactions, similar with results we obtained in another study with respect to the blood flow in the vessels of chorioallantoic membrane. It can be concluded that probably in late-term bird embryos $\mathrm{NO}$ acts as a mediator of the neuronal cold pathway in the anterior hypothalamus.

\section{Acknowledgment}

This study was supported by grants of the Deutsche Forschungsgemeinschaft (436 WER 17/7/04, 17/3/05, and $17 / 5 / 06)$.

\section{References}

[1] B. Tzschentke and A. Plagemann, "Imprinting and critical periods in early development," World's Poultry Science Journal, vol. 62, no. 4, pp. 626-726, 2006.

[2] M. Szyf, P. McGowan, and M. J. Meaney, "The social environment and the epigenome," Environmental and Molecular Mutagenesis, vol. 49, no. 1, pp. 46-60, 2008.

[3] A. Nott and A. Riccio, "Nitric oxide-mediated epigenetic mechanisms in developing neurons," Cell Cycle, vol. 8, no. 5, pp. 725-730, 2009.

[4] J. Garthwaite and C. L. Boulton, "Nitric oxide signaling in the central nervous system," Annual Review of Physiology, vol. 57, pp. 683-706, 1995.

[5] J. Garthwaite, "Concepts of neural nitric oxide-mediated transmission," European Journal of Neuroscience, vol. 27, no. 11, pp. 2783-2802, 2008.

[6] C. L. M. Bon and J. Garthwaite, "On the role of nitric oxide in hippocampal long-term potentiation," Journal of Neuroscience, vol. 23, no. 5, pp. 1941-1948, 2003.

[7] S. Liu, M. Fa, I. Ninan, F. Trinchese, W. Dauer, and O. Arancio, " $\alpha$-Synuclein involvement in hippocampal synaptic plasticity: role of NO, cGMP, cGK and CaMKII," European Journal of Neuroscience, vol. 25, no. 12, pp. 3583-3596, 2007.

[8] N. Majlessi, S. Choopani, T. Bozorgmehr, and Z. Azizi, "Involvement of hippocampal nitric oxide in spatial learning in the rat," Neurobiology of Learning and Memory, vol. 90, no. 2, pp. 413-419, 2008.

[9] E. Fino, V. Paille, J. M. Deniau, and L. Venance, "Asymmetric spike-timing dependent plasticity of striatal nitric oxidesynthase interneurons," Neuroscience, vol. 160, no. 4, pp. 744754, 2009.
[10] J. Zhang and S. H. Snyder, "Nitric oxide in the nervous system," Annual Review of Pharmacology and Toxicology, vol. 35, pp. 213-233, 1995.

[11] H. C. Pape and R. Mager, "Nitric oxide controls oscillatory activity in thalamocortical neurons," Neuron, vol. 9, no. 3, pp. 441-448, 1992.

[12] W. F. Taylor and V. S. Bishop, "A role for nitric oxide in active thermoregulatory vasodilation," American Journal of Physiology, vol. 264, no. 5, pp. H1355-H1359, 1993.

[13] V. I. Dunai and A. V. Gourine, "Effect of the NO synthase inhibitor, L-NAME, on body temperature in birds in different periods of postnatal ontogenesis," in Recent Advances in Thermal Biology, V. N. Guorine, Ed., pp. 18-19, Minsk, 1999.

[14] S. S. Malik and J. E. Fewell, "Thermoregulation in rats during early postnatal maturation: importance of nitric oxide," American Journal of Physiology, vol. 285, no. 6, pp. R1366R1372, 2003.

[15] D. H. S. Pereira, L. G. S. Branco, E. C. Cárnio, and R. C. H. Barros, "nNOS is involved in behavioral thermoregulation of newborn rats during hypoxia," Physiology and Behavior, vol. 89, no. 5, pp. 681-686, 2006.

[16] A. C. Coleone, K. A. A. Torres, E. C. Carnio et al., "Role of brain nitric oxide in the thermoregulation of broiler chicks," Comparative Biochemistry and Physiology, vol. 154, no. 2, pp. 204-210, 2009.

[17] T. Hübschle, I. Küchenmeister, and R. Gerstberger, "Central action of nitric oxide in the saltwater-acclimated duck: modulation of extrarenal sodium excretion and vasotocin release," Brain Research, vol. 825, no. 1-2, pp. 22-35, 1999.

[18] H. Schwimmer, R. Gerstberger, and M. Horowitz, "Heat acclimation affects the neuromodulatory role of AngII and nitric oxide during combined heat and hypohydration stress," Molecular Brain Research, vol. 130, no. 1-2, pp. 95-108, 2004.

[19] S. Amir, E. De Blasio, and A. M. English, "N(G)-Monomethyl$\mathrm{L}$-arginine co-injection attenuates the thermogenic and hyperthermic effects of $E_{2}$ prostaglandin microinjection into the anterior hypothalamic preoptic area in rats," Brain Research, vol. 556, no. 1, pp. 157-160, 1991.

[20] A. V. Gourine, "Role of nitric oxide in lipopolysaccharideinduced fever in conscious rabbits," The Journal of Physiology, vol. 475, p. 28, 1994.

[21] Y. H. Choi, M. Furuse, J. I. Okumura, and D. M. Denbow, "Nitric oxide controls feeding behavior in the chicken," Brain Research, vol. 654, no. 1, pp. 163-166, 1994.

[22] R. Vozzo, G. A. Wittert, I. M. Chapman et al., "Evidence that nitric oxide stimulates feeding in the marsupial Sminthopsis crassicaudata," Comparative Biochemistry and Physiology, vol. 123, no. 2, pp. 145-151, 1999.

[23] S. J. Yang and D. M. Denbow, "Interaction of leptin and nitric oxide on food intake in broilers and Leghorns," Physiology and Behavior, vol. 92, no. 4, pp. 651-657, 2007.

[24] M. S. I. Khan, T. Tachibana, Y. Hasebe, N. Masuda, and H. Ueda, "Peripheral or central administration of nitric oxide synthase inhibitor affects feeding behavior in chicks," Comparative Biochemistry and Physiology, vol. 148, no. 2, pp. 458-462, 2007.

[25] M. S. I. Khan, Y. Nakano, T. Tachibana, and H. Ueda, "Nitric oxide synthase inhibitor attenuates the anorexigenic effect of corticotropin-releasing hormone in neonatal chicks," Comparative Biochemistry and Physiology, vol. 149, no. 3, pp. 325-329, 2008.

[26] C. J. Sadler and J. P. H. Wilding, "Reduced ventromedial hypothalamic neuronal nitric oxide synthase and increased sensitivity to NOS inhibition in dietary obese rats: further 
evidence of a role for nitric oxide in the regulation of energy balance," Brain Research, vol. 1016, no. 2, pp. 222-228, 2004.

[27] T. Horn, P. M. Smith, B. E. McLaughlin et al., "Nitric oxide actions in paraventricular nucleus: cardiovascular and neurochemical implications," American Journal of Physiology, vol. 266, no. 1, pp. R306-R313, 1994.

[28] B. C. W. Groenendijk, B. P. Hierck, A. C. Gittenberger-De Groot, and R. E. Poelmann, "Development-related changes in the expression of shear stress responsive genes KLF-2, ET-1, and NOS-3 in the developing cardiovascular system of chicken embryos," Developmental Dynamics, vol. 230, no. 1, pp. 57-68, 2004.

[29] S. Pyner, "Neurochemistry of the paraventricular nucleus of the hypothalamus: implications for cardiovascular regulation," Journal of Chemical Neuroanatomy, vol. 38, no. 3, pp. 197-208, 2009.

[30] S. Eriksson, H. Hjelmqvist, R. Keil, and R. Gerstberger, "Central application of a nitric oxide donor activates heat defense in the rabbit," Brain Research, vol. 774, no. 1-2, pp. 269-273, 1997.

[31] G. Bruning, "Localization of NADPH-diaphorase in the brain of the chicken," Journal of Comparative Neurology, vol. 334, no. 2, pp. 192-208, 1993.

[32] C. M. Montagnese and A. Csillag, "Comparative distribution of NADPH-diaphorase activity and tyrosine hydroxylase immunoreactivity in the diencephalon and mesencephalon of the domestic chicken (Gallus domesticus)," Anatomy and Embryology, vol. 193, no. 5, pp. 427-439, 1996.

[33] M. Nichelmann and B. Tzschentke, "Ontogeny of thermoregulation in precocial birds," Comparative Biochemistry and Physiology, vol. 131, no. 4, pp. 751-763, 2002.

[34] B. Tzschentke and D. Basta, "Development of hypothalamic neuronal thermosensitivity in birds during the perinatal period," Journal of Thermal Biology, vol. 25, no. 1-2, pp. 119$123,2000$.

[35] B. Tzschentke and D. Basta, "Early development of neuronal hypothalamic thermosensitivity in birds: influence of epigenetic temperature adaptation," Comparative Biochemistry and Physiology, vol. 131, no. 4, pp. 825-832, 2002.

[36] B. Tzschentke, "Attainment of thermoregulation as affected by environmental factors," Poultry Science, vol. 86, no. 5, pp. 1025-1036, 2007.

[37] B. Tzschentke and M. Rumpf, "Embryonic development of endothermy," Respiratory Physiology and Neurobiology, vol. 178, no. 1, pp. 97-107, 2011.

[38] B. Tzschentke, D. Basta, O. Janke, and I. Maier, "Characteristics of early development of body functions and epigenetic adaptation to the environment in poultry: focused on development of central nervous mechanisms," Avian and Poultry Biology Reviews, vol. 15, no. 3-4, pp. 107-118, 2004.

[39] O. Janke and B. Tzschentke, "Long-lasting effect of changes in incubation temperature on heat stress induced neuronal hypothalamic c-Fos expression in chickens. Special Issue: early development and epigenetic programming of body functions in birds (Ed. Tzschentke, B.)," The Open Ornithology Journal, vol. 3, pp. 150-155, 2010.

[40] G. C. Whittow and H. Tazawa, "The early development of thermoregulation in birds," Physiological Zoology, vol. 64, no. 6, pp. 1371-1390, 1991.

[41] H. Tazawa and G. C. Whittow, "Incubation physiology," in Sturkey's Avian Physiology, pp. 617-634, Academic Press, New York, NY, USA, 5th edition, 2000.

[42] U. Scherer Singler, S. R. Vincent, H. Kimura, and E. G. McGeer, "Demonstration of a unique population of neurons with NADPH-diaphorase histochemistry," Journal of Neuroscience Methods, vol. 9, no. 3, pp. 229-234, 1983.

[43] B. T. Hope and S. R. Vincent, "Histochemical characterization of neuronal NADPH-diaphorase," Journal of Histochemistry and Cytochemistry, vol. 37, no. 5, pp. 653-661, 1989.

[44] T. Matsumoto, M. Nakane, J. S. Pollock, J. E. Kuk, and U. Forstermann, "A correlation between soluble brain nitric oxide synthase and NADPH-diaphorase activity is only seen after exposure of the tissue to fixative," Neuroscience Letters, vol. 155, no. 1, pp. 61-64, 1993.

[45] W. J. Kuenzel and A. van Tienhoven, "Nomenclature and location of avian hypothalamic nuclei and associated circumventricular organs," Journal of Comparative Neurology, vol. 206, no. 3, pp. 293-313, 1982.

[46] W. J. Kuenzel and M. Masson, A Stereotaxic Atlas of the Brain of the Chick (Gallus domesticus), The John Hopkins University Press, Baltimore, Md, USA, 1998.

[47] B. Tzschentke, "Monitoring the development of thermoregulation in poultry embryos and its influence by incubation temperature," Computers and Electronics in Agriculture, vol. 64, no. 1, pp. 61-71, 2008.

[48] M. Nichelmann and B. Tzschentke, "Thermoregulation in precocial avian embryos," Ornis Fennica, vol. 76, no. 4, pp. 177-187, 1999.

[49] O. Janke, B. Tzschentke, J. Höchel, and M. Nichelmann, "Metabolic responses of chicken and muscovy duck embryos to high incubation temperatures," Comparative Biochemistry and Physiology, vol. 131, no. 4, pp. 741-750, 2002.

[50] M. Nichelmann, A. Burmeister, O. Janke, J. Höchel, and B. Tzschentke, "Avian embryonic thermoregulation: role of Q10 in interpretation of endothermic reactions," Journal of Thermal Biology, vol. 23, no. 6, pp. 369-376, 1998.

[51] B. Loh, I. Maier, A. Winar, O. Janke, and B. Tzschentke, "Prenatal development of epigenetic adaptation processes in poultry: changes in metabolic and neuronal thermoregulatory mechanisms," Avian and Poultry Biology Reviews, vol. 15, no. 3-4, pp. 119-128, 2004.

[52] T. Nakashima, F. K. Pierau, E. Simon, and T. Hori, "Comparison between hypothalamic thermoresponsive neurons from duck and rat slices," Pflugers Archiv European Journal of Physiology, vol. 409, no. 3, pp. 236-243, 1987.

[53] H. A. Schmid, L. Jansky, and F. -K. Pierau, "Temperature sensitivity of neurons in slices of the rat PO/AH area: effect of bombesin and substance P," American Journal of Physiology, vol. 264, no. 2, pp. R449-R455, 1993.

[54] B. Tzschentke and I. Halle, "Influence of temperature stimulation during the last 4 days of incubation on secondary sex ratio and later performance in male and female broiler chicks," British Poultry Science, vol. 50, no. 5, pp. 634-640, 2009.

[55] B. Tzschentke and M. Nichelmann, "Development of avian thermoregulatory system during the early postnatal period: development of the thermoregulatory set-point," Ornis Fennica, vol. 76, no. 4, pp. 189-198, 1999.

[56] T. M. Gilbert and C. M. Blatteis, "Hypothalamic thermoregulatory pathways in the rat," Journal of Applied Physiology Respiratory Environmental and Exercise Physiology, vol. 43, no. 5, pp. 770-777, 1977.

[57] K. Kanosue, M. Yanase-Fujiwara, and T. Hosono, "Hypothalamic network for thermoregulatory vasomotor control," American Journal of Physiology, vol. 267, no. 1, pp. R283-R288, 1994. 
[58] K. Kanosue, Zhang Yi Hong, M. Yanase-Fujiwara, and T. Hosono, "Hypothalamic network for thermoregulatory shivering," American Journal of Physiology, vol. 267, no. 1, pp. R275-R282, 1994.

[59] B. J. Oldfield, M. E. Giles, A. Watson, C. Anderson, L. M. Colvill, and M. J. McKinley, "The neurochemical characterisation of hypothalamic pathways projecting polysynaptically to brown adipose tissue in the rat," Neuroscience, vol. 110, no. 3, pp. 515-526, 2002.

[60] P. R. Kamerman, H. P. Laburn, and D. Mitchell, "Inhibitors of nitric oxide synthesis block cold-induced thermogenesis in rats," Canadian Journal of Physiology and Pharmacology, vol. 81, no. 8, pp. 834-838, 2003.

[61] A. C. R. Lacerda, U. Marubayashi, and C. C. Coimbra, "Nitric oxide pathway is an important modulator of heat loss in rats during exercise," Brain Research Bulletin, vol. 67, no. 1-2, pp. 110-116, 2005.

[62] A. A. Steiner, J. Antunes-Rodrigues, S. M. McCann, and L. G. S. Branco, "Antipyretic role of the NO-cGMP pathway in the anteroventral preoptic region of the rat brain," American Journal of Physiology, vol. 282, no. 2, pp. R584-R593, 2002.

[63] A. Ar, O. Ifergan, A. Feldman, L. Zelik, and A. Reizis, "Possible role of nitric oxide emission from bird embryos," Avian and Poultry Biology Reviews, vol. 15, no. 3-4, pp. 105-106, 2004. 

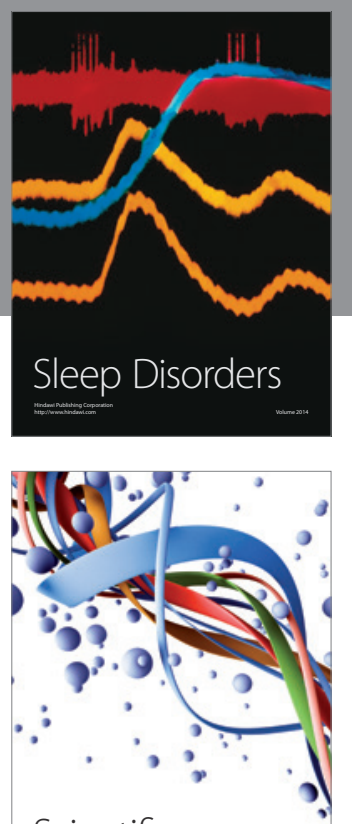

Scientifica
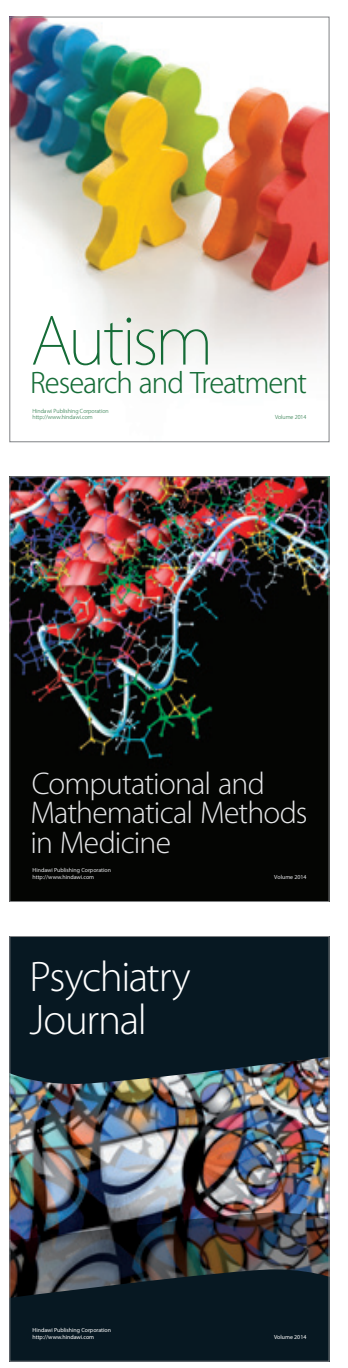
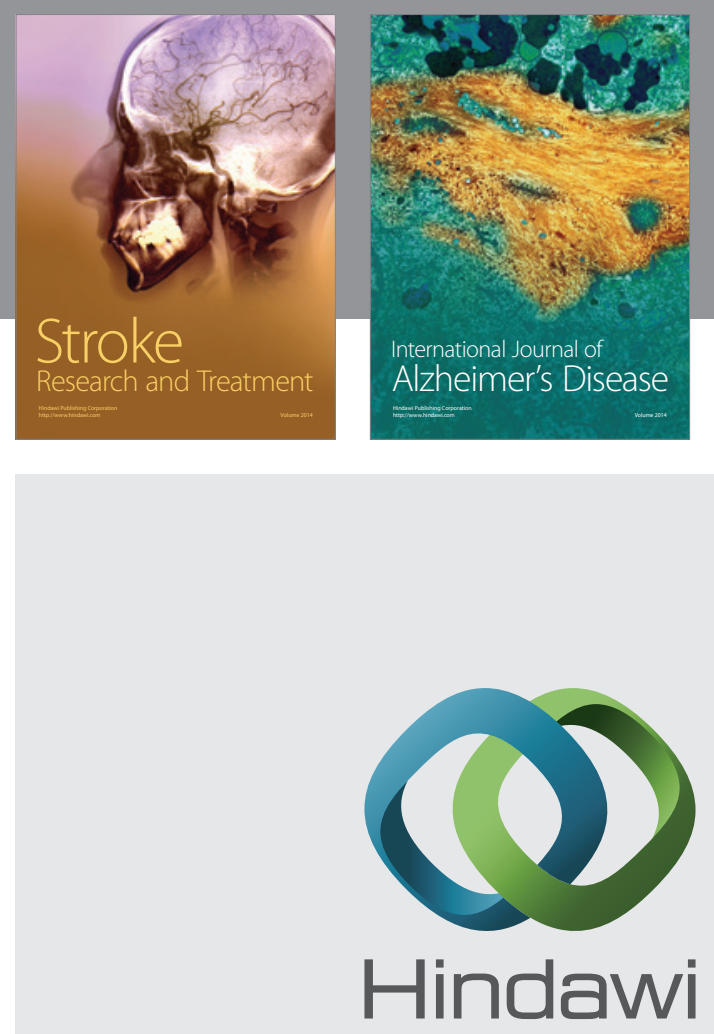

Submit your manuscripts at

http://www.hindawi.com
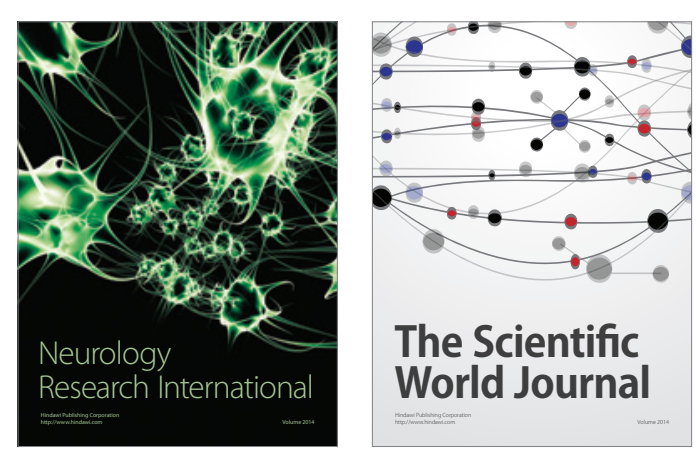

The Scientific World Journal

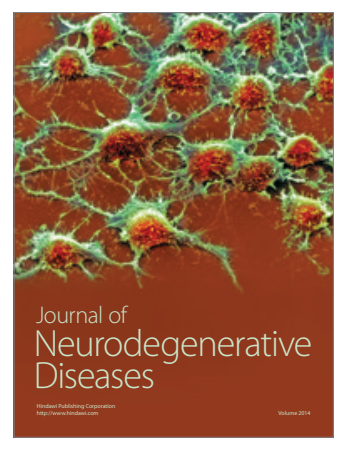

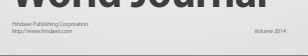

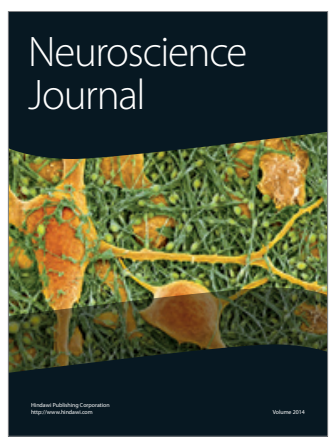

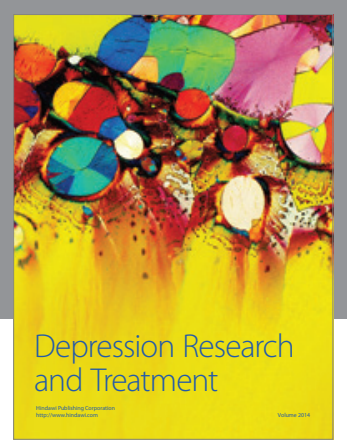
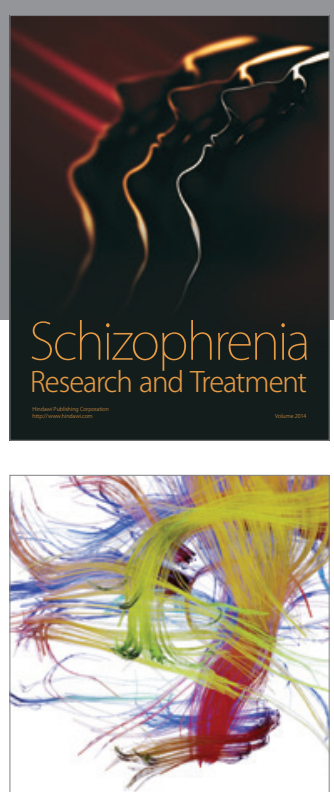

Brain Science

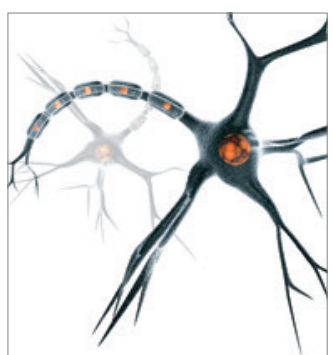

Neural Plasticity
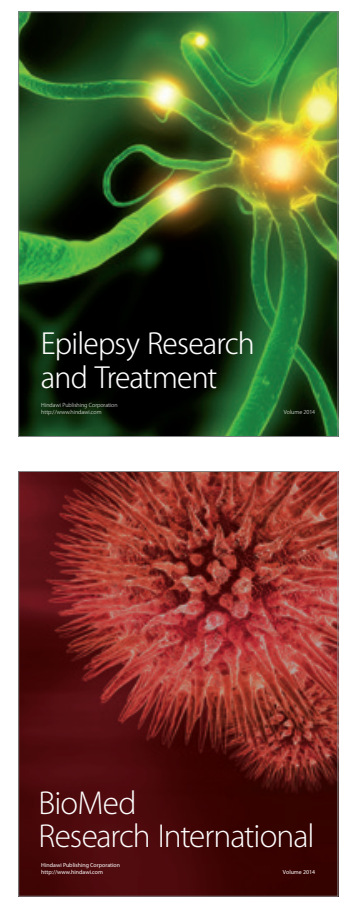

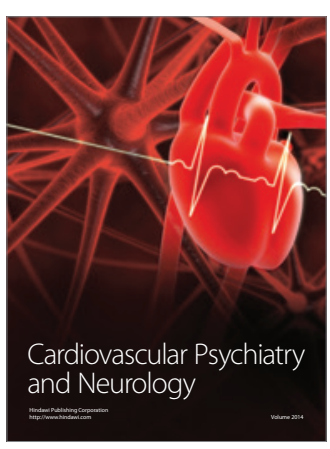

Parkinson's

Disease
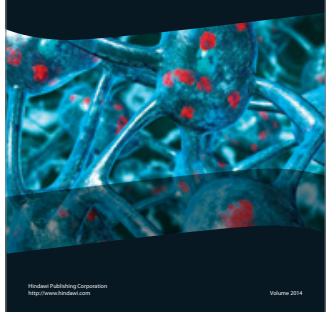